\title{
Eating disorders and obesity (ED\&O) in the COVID-19 storm
}

\author{
Patrizia Todisco $^{1} \cdot$ Lorenzo M. Donini $^{2}$
}

Received: 20 May 2020 / Accepted: 21 May 2020 / Published online: 1 June 2020

(c) Springer Nature Switzerland AG 2020

\section{The COVID-19 storm}

On 9 January 2020, the China CDC reported that a novel coronavirus (SARS-CoV-2) had been detected as the causative agent of the respiratory disease later named COVID19. Since then COVID-19 has affected a large part of the population of the planet in a very rapid and violent way [1].

It has put a strain on healthcare systems that have had difficulties in managing the emergency in the acute phase and the economic system that has found itself facing a sharp and massive slowdown in trade and, therefore, in production. This meant a significant number of infected people, sick people and death [WHO data (Health Emergency Dashboard) on 3rd of May 2020 report: 3,272,202 confirmed cases worldwide since the start of the outbreak; 230,104 deaths; 208 countries, areas or territories with cases], but also a significant number of unemployed (according to Goldman Sachs analysts, the unemployment rate in the Eurozone could rise to $11 \%$ by mid-year) in relation to the lockdown of many economic activities and a widespread economic hardship that promises to be even more serious in the coming months.

The response of the various countries to the emergency has been different with variations in admissions to hospitals or to intensive care units [probably due to different hospitalization guidelines and thresholds, hospital capacities, as well as to the risk factors (e.g. age, comorbidity) among the infected persons [www.euro.who.int/_data/ assets/pdf_file/0006/437469/TG2-CreatingSurgeAcuteIC Ucapacity-eng.pdf], with different pharmacological protocols (which have combined differently from country to country, from region to region, antimalarial and antiviral

Patrizia Todisco

patrizia.todisco1964@gmail.com

1 Eating Disorders Unit, Casa Di Cura "Villa Margherita", Arcugnano (Vicenza), Italy

2 Department of Experimental Medicine, Sapienza University, Rome, Italy drugs, corticosteroids, antibiotics, oxygen therapy, anticoagulant medications, ...), experimenting new interventions often borrowed from other situations [biologics, interferon, antiretroviral medications, ...: 382 trials were registered between 23rd January and 8th March 2020 on the WHO's International Clinical Trials Registry Platform (ICTRP), https://www.cebm.net/covid-19/registered-trials-and-analy sis/] while waiting for a vaccine and specific therapy (immunotherapy ?).

During lockdown, there were different problems of public health (not only those for COVID-19) which went second. Most emergency units, and in general many units in several hospitals and settings, were filled by patients affected by SARS-CoV-2 infection and there was no place to give assistance to other types of patients (above all those in critical conditions, leading to additional deaths). Besides, most subjects didn't go to the Emergency Department because they feared to infect themselves, in this way, delaying diagnosis and outcome of acute conditions. For instance, in U.S. hospitals, there was a decrease of approximately $39 \%$ in the numbers of patients who received evaluations for acute stroke between the pre-pandemic epoch and the early-pandemic epoch [2]; in Italy, official hospital statistics in the period March 1-27, 2020, show substantial decreases-ranging from 73 to $88 \%$ - in paediatric emergency department visits compared with the same time period in 2019 and 2018, and family paediatricians widely report a considerable reduction in clinic visits [3].

COVID-19 has painfully exposed the existing and persisting health inequalities in our societies and will have the heaviest impact on the lives of people living in deprivation or facing difficult socio-economic circumstances. These people are more likely to suffer of chronic illnesses and mental disorders and in this difficult period, they could have decided not to refer to the healthcare because of lack of money. Epidemics and economic crises can have a disproportionate impact on the most vulnerable segments of the population, which can trigger worsening inequality.

In all countries, even if with different intensities, the socalled social distancing with travel restriction policies (https 
://www.cdc.gov/coronavirus/2019-ncov/prevent-gettingsick/social-distancing.html) and the lockdown of non-essential economic activities represented the common approach to contrast the spread of the virus. Human beings, always accustomed to interact socially, suddenly found themselves being more (or completely) alone, having to keep their distance from relatives, friends, and colleagues with obvious and inevitable psychological consequences.

All three pillars that define health status as a "physical, mental and social well-being" (Preamble to the Constitution of WHO as adopted by the International Health Conference, New York, 19 June-22 July 1946-Official Records of WHO, no. 2, p. 100) have been affected by the COVID-19 pandemic.

\section{ED\&O patients in the COVID-19 storm}

Patients with eating disorders and/or obesity (ED\&O) represent a group of subjects, which, beyond the medical or psychological specificities typical of each disease, have in common a significant frailty. Similarly to elderly subjects, ED\&O patients are characterised by decreased resilience and physiological reserves across multiple organ systems, arising from cumulative comorbid conditions. ED\&O have: 1. a psychological discomfort linked to the disease and the psychiatric consequences and comorbidities; 2 . medical problems as a result of eating behaviors and metabolic imbalances; 3. social problems as a consequence of the disorder, of the physical disability, of belonging often to problematic families and, in particular for people with obesity, to economically poorer social classes and with a lower cultural level.

It is, therefore, likely that COVID-19 may have affected subjects with ED\&O more heavily from a medical point of view but the data in this sense are often conflicting. A greater viral pathogenicity of the severe acute respiratory syndrome linked to COVID-19, as a consequence of a diminished immune function and/or of a maladaptive cycle of local inflammation in individuals with obesity, was suggested by several authors [4] but some others didn't confirm this hypothesis [5]. Subjects with malnutrition (undernutrition) may be particularly vulnerable to COVID-19, but the impact on patients with anorexia nervosa is unclear since these patients have shown a high level of resilience to viral illnesses [6].

The outbreaks of COVID-19 pandemic, and in particular economic difficulties and social distancing actually have made access to treatment more problematic. In this situation, the diagnosis could have been delayed and the beginning of treatment too, the healthcare providers may have had more difficulties in assessing the severity of ED\&O symptoms and implementing the appropriate treatment, due to the limitation to only urgent visits and to inpatient treatments in many healthcare settings. To reach people with ED\&O may have been more challenging, since attention has been focused on managing the COVID-19 emergency. Psychotherapies have been transferred to videoconferencing systems that are probably not suitable for all patients (and perhaps not even for all psychotherapists) and this change in setting can possibly have affected the therapeutic alliance and the treatment outcome. The economic difficulties may have hindered the access to diagnostics and treatments in subjects who are almost always characterized by important comorbidities and who need intense and constant care [7-9].

Moreover, patients with ED\&O, who are already isolated both emotionally and physically, may have experienced a worsening of symptoms connected to living alone or in close contact with their families. The need for control may have been even more exacerbated by the unpredictability of the situation (e.g. in restrictive anorexia), whereas the greater ease of access to food while staying at home may have had negative consequences in patients with binge eating (e.g. bulimia nervosa and binge eating disorder). The greater difficulty in implementing compensatory behaviours (physical activity, purging behaviors, ...) may have affected some patients while complexity in emotional regulation may have triggered binge eating episodes. These situations may have been further exasperated by the constant contact with family members who are also worried, conflicting and controlling $[7,10]$.

Finally, social media posts, which warned against the risk of weight gain during social distancing due to vulnerability to overeating and sedentary behavior, may have somehow represented a form of weight stigma. Most of these posts [the so-called "quarantine-15" that refers to the risk of $15-\mathrm{lb}$ weight gain (around $7 \mathrm{~kg}$ ) during self-isolation] have directly or indirectly elicited common stereotypes suggesting that people with obesity are lazy, shabby and without self-control while promoting unrealistic thin ideals and extreme weight control practices. The internalisation of negative weight stereotypes may have harmful effects, since it weakens selfefficacy and confidence in personal ability to achieve an effective control on eating behaviour [11] and this is even more important in ED\&O patients or in people at risk for these disorders.

Besides the consequences that this pandemic is having on public health, different papers alert about a possible higher prevalence of post-traumatic stress disorders (PTSD) after COVID-19 within the general population. PTSD may be related to the fear of contagion or dying, to surviving to COVID-19, to the death of family members or close friends; they may be highlighted by anxiety, sadness, anger and loneliness related to social isolation. During the 2003 SARS outbreak, people who had been quarantined, or who worked in high-risk locations such as SARS wards, or who had friends or close relatives who contracted SARS, were 
much more likely to have mental health problems [12]. This means that we have to consider not only the psychological and medical situation of ED\&O patients but also new posttraumatic outbreaks [6, 13]. In particular, the mechanisms of emergence of these disorders and their interrelationships remain to be studied considering in particular the context of confinement [14].

\section{The quiet after the storm}

When trying to return to normality, the problem arises of how to do it and in particular, how rapidly to do it. If in the first phase of emergency, the aspects of contrast to the spread of the virus have (probably rightly) prevailed, in this second phase, the other aspects related to the social distancing and to the risk of impoverishment must have a fair weight.

Policy makers are, therefore, called to consider the balance between these different factors and to find the right equilibrium between them when they plan to return to normal activities. Mass isolation during 2003 SARS outbreak caused an increase of psychological disorders and the predicting factor with the highest correlation was income reduction $(\mathrm{OR}=25.0)$ in comparison to other factors (e.g. gender, range of activities, eating restrictions, restrictions in going out, disinfection of clothing, and infection control which had an $\mathrm{OR}=3.2,5.5,3.9,3.2,0.2$, and 0.1 , respectively) [13].

The researchers and the scientific societies dealing with ED\&O will have to promote research to correctly evaluate the impact on the psychological and clinical status of social distancing and to verify if all the diagnostic and therapeutic instruments that have been tuned before the COVID-19 pandemic maintain their validity [8]. They will also have to give operational indications to healthcare professionals (HCP) establishing guidelines for taking charge of these further problems in subjects already clinically and psychologically engaged. Finally, they have to promote training to make HCP more capable to prepare ED\&O patients and their families to changes in the environment, in promoting/supervising self-help strategies that have shown to be effective in some cases, and in using teletherapy in particular for assessing patients with ED\&O who are also coping with the stress of isolation [14].

The HCP will have to take into account the additional psychological, functional and, last but not least, economic difficulties that the patients might have. They will also be aware of the possibility that ED\&O may arise in their incidence and prepared to make early diagnosis and to deliver prevention protocol above all to population at risk for ED\&O development. Some attempts to define an effective strategy in the management of ED have been made. Recommendations for patients, caregivers and therapists have been established, looking at eating- and non-eating-specific situations, differentiating between maladaptive and adaptive strategies, defining coping strategies and positive/alternative thinking [7]. Moreover, different institutions have proposed virtual support instruments to help people and caregivers to deal with ED\&O.

(e.g. https://www.healthline.com/health/mental-health/5reminders-for-eating-disorder-survivors-during-the-covid -19-outbreak).

It will probably take time to fully evaluate the impact that COVID-19 has had on the health status of patients with ED\&O, but it is important that the reflection begins immediately and that information is collected from now on to better manage the emergency exit phase and the periods following this.

\section{Compliance with ethical standards}

Conflict of interest The authors declare that they have no conflict of interest.

Ethical approval The article is an Editorial. It was not funded and did not need ethical approval.

Informed consent Informed consent is not required.

\section{References}

1. Onder G, Rezza G, Brusaferro S (2020) Case-fatality rate and characteristics of patients dying in relation to COVID-19 in Italy. JAMA. https://doi.org/10.1001/jama.2020.4683 (published online ahead of print, $2020 \mathrm{Mar} 23$ )

2. Kansagra AP, Goyal MS, Hamilton S, Albers GW (2020) Collateral effect of Covid-19 on stroke evaluation in the United States. N Engl J Med. https://doi.org/10.1056/NEJMc2014816

3. Lazzerini M, Barbi E, Apicella A, Marchetti F, Cardinale F, Trobia G (2020) Delayed access or provision of care in Italy resulting from fear of COVID-19. Lancet Child Adolesc Health 4(5):e10-e11

4. Carter SJ, Baranauskas MN, Fly AD (2020) Considerations for obesity, vitamin D, and physical activity amidst the COVID19 pandemic. Obesity (Silver Spring). https://doi.org/10.1002/ oby. 22838

5. Jose RJ, Manuel A (2020) Does COVID-19 disprove the obesity paradox in ARDS? Obesity (Silver Spring). https://doi. org/10.1002/oby.22835

6. Touyz S, Lacey H, Hay P (2020) Eating disorders in the time of COVID-19. J Eat Disord 8:19. https://doi.org/10.1186/s4033 7-020-00295-3 (eCollection 2020)

7. Fernández-Aranda F, Casas M, Claes L, Bryan DC, Favaro A, Granero R, Gudiol C, Jiménez-Murcia S, Karwautz A, Le Grange D, Menchón JM, Tchanturia K, Treasure J (2020) COVID-19 and implications for eating disorders. Eur Eat Disord Rev 28(3):239 245. https://doi.org/10.1002/erv.2738

8. Davis C, Ng KC, Oh JY, Baeg A, Rajasegaran K, Chew CSE (2020) Caring for children and adolescents with eating disorders in the current coronavirus 19 pandemic: a singapore perspective. $\mathrm{J}$ Adolesc Health. https://doi.org/10.1016/j.jadohealth.2020.03.037 
9. Sproch LE, Anderson KP (2019) Clinician-delivered teletherapy for eating disorders. Psychiatr Clin North Am 42(2):243-252. https://doi.org/10.1016/j.psc.2019.01.008

10. Weissman RS, Bauer S, Thomas JJ (2020) Access to evidencebased care for eating disorders during the COVID-19 crisis. Int J Eat Disord. https://doi.org/10.1002/eat.23279

11. Pearl RL (2020) Weight stigma and the "Quarantine-15". Obesity (Silver Spring). https://doi.org/10.1002/oby.22850

12. Ghebreyesus TA (2020) Addressing mental health needs: an integral part of COVID-19 response. World Psych 19(2):129-130

13. Liu N, Zhang F, Wei C, Jia Y, Shang Z, Sun L, Liu W (2020) Prevalence and predictors of PTSS during COVID-19 outbreak in China hardest-hit areas: gender differences matter. Psychiatry Res 287:112921. https://doi.org/10.1016/j.psychres.2020.112921

14. Mengin A, Allé MC, Rolling J, Ligier F, Schroder C, Lalanne L, Berna F, Jardri R, Vaiva G, Geoffroy PA, Brunault P, Thibaut F, Chevance A, Giersch A (2020) Psychopathological consequences of confinement. Encephale. https://doi.org/10.1016/j. encep.2020.04.007
15. Mihashi M, Otsubo Y, Yinjuan X, Nagatomi K, Hoshiko M, Ishitake T (2009) Predictive factors of psychological disorder development during recovery following SARS outbreak. Health Psychol 28(1):91-100. https://doi.org/10.1037/a0013674

16. Waller G, Pugh M, Mulkens S, Moore E, Mountford VA, Carter J, Wicksteed A, Maharaj A, Wade TD, Wisniewski L, Farrell NR, Raykos B, Jorgensen S, Evans J, Thomas JJ, Osenk I, Paddock C, Bohrer B, Anderson K, Turner H, Hildebrandt T, Xanidis N, Smit V (2020) Cognitive-behavioral therapy in the time of coronavirus: clinician tips for working with eating disorders via telehealth when face-to-face meetings are not possible. Int J Eat Disord. https://doi.org/10.1002/eat.23289

Publisher's Note Springer Nature remains neutral with regard to jurisdictional claims in published maps and institutional affiliations. 